\title{
Copy number variation profiling in pharmacogenetics CYP-450 and GST genes in Colombian population
}

Brian Ramírez ${ }^{\dagger}$, María José Niño-Orrego ${ }^{\dagger}$, Daniel Cárdenas, Kevin Enrique Ariza, Karol Quintero, Nora Constanza Contreras Bravo(D, Caroll Tamayo-Agudelo, María Alejandra González, Paul Laissue@ and Dora Janeth Fonseca Mendoza ${ }^{*}$ (D)

\begin{abstract}
Background: Copy Number variation (CNVs) in genes related to drug absorption, distribution, metabolism and excretion (ADME) are relevant in the interindividual variability of drug response. Studies of the CNVs in ADME genes in Latin America population are lacking. The objective of the study was to identify the genetic variability of CNVs in CYP-450 and GST genes in a subgroup of individuals of Colombian origin.

Methods: Genomic DNA was isolated from 123 healthy individuals from a Colombian population. Multiplex Ligation-Dependent Probe Amplification (MLPA) was performed for the identification of CNVs in 40 genomic regions of 11 CYP-450 and 3 GST genes. The genetic variability, allelic and genotypic frequencies were analyzed.

Results: We found that 13 out of 14 genes had CNVs: 5 (35.7\%) exhibited deletions and duplications, while 8 (57.1\%) presented either deletions or duplications.. 33.3\% of individuals carried deletions and duplications while $49.6 \%$ had a unique type of CNV (deletion or duplication). The allelic frequencies of the CYP and GST genes were 0 to $47.6 \%$ (allele null), 0 to $17.5 \%$ (duplicated alleles) and 37 to 100\% (normal alleles).

Conclusions: Our results describe, for the first time, the genomic profile of CNVs in a subgroup of Colombian population in GST and CYP-450 genes. GST genes indicated greater genetic variability than CYP-450 genes. The data obtained contributes to the knowledge of genetic profiles in Latin American subgroups. Although the clinical relevance of CNVs has not been fully established, it is a valuable source of pharmacogenetic variability data with potential involvement in the response to medications.
\end{abstract}

Keywords: DNA copy number variation, Allele frequency, Personalized medicine, Pharmacogenomics,DNA

\section{Background}

Interindividual variability response to drugs has been associated with multiple genetic and environmental factors [1]. Genetic variants in genes encoding proteins related to drug absorption, distribution, metabolism and excretion (ADME) have shown to impact on pharmacokinetics, pharmacodynamics efficacy and safety [2-4]. In view of the variation of pharmacogenes relevant in clinical practice, the FDA (US Food and Drug Administration)

\footnotetext{
* Correspondence: dora.fonseca@urosario.edu.co

†Brian Ramírez and María José Niño-Orrego contributed equally to this work. GENIUROS Research Group, Center For Research in Genetics and Genomics CIGGUR, School of Medicine and Health Sciences, Universidad Del Rosario, Carrera 24 Nº 63C-69, CP 112111, Bogotá DC, Colombia
}

and EMA (European Medicines Agency) have recognized the benefit of genotyping some validated biomarkers for the identification of cases at risk of potential toxicity or therapeutic failure. In this context, genetic analysis facilitates the selection of a safer and more effective pharmacological management for each patient.

Despite the fact that Single Nucleotide Variants (SNVs) are the most widely studied variants, there has been a recent recognition in the influence of CNVs on interindividual differences in drug medication response [5]. It has been estimated that around $12 \%$ of the human genome contains CNVs, which are defined as duplications or deletions of DNA segments from $1 \mathrm{~Kb}$ to $3 \mathrm{~Kb}[1,6]$. It has been determined that several pharmacogenes of

(c) The Author(s). 2019 Open Access This article is distributed under the terms of the Creative Commons Attribution 4.0 International License (http://creativecommons.org/licenses/by/4.0/), which permits unrestricted use, distribution, and 
clinical relevance (e.g. CYP2D6, GSTT1, GSTM1, SULT1A1, CYP2A6, and UGT2B17) contain CNVs associated to the variation of enzymatic activity observed among different populations. CYP2D6 and CYP2A6 constitute coding genes for Phase I metabolism enzymes and display the greatest number of reported CNVs [4]. Regarding the Phase II metabolism enzymes, CNVs in the glutathione transferase enzymes and sulfotransferases have been reported. These genes are involved in drug metabolism and detoxification of xenobiotics [1, 7-9]. In the Latin-American population there is a notable absence of genetic studies, and with exception of CYP2D6 there is a gap regarding frequency of drug-related CNVs [10]. Analyses using autosomal and sexual markers performed in the Latin American population have indicated a great variation in the influence of African / European and Native ancestry between individuals and geographic regions. The analysis of SNPs in more than 6000 individuals in 5 Latin American countries has estimated that the highest proportion of African ancestry occurs in Brazil (9.3\%) and Colombia (9.6\%) (with ranges for other countries between 4.6 and 9.6); the native in Peru (64.8\%) (ranges of 12.1 to 64.8\%) and the European in Brazil (78.6\%) and Colombia (61.2\%) (ranges of 30.6 to78.6\%) [11]. These findings reflect a high heterogeneity in the structure of these populations [10,11].

Although clinical relevance still needs to be established, CNVs play a clear role in drug-related genes as they alter metabolism and therapeutic response $[1,4,12,13]$.

The present study analyzed 40 genomic regions of GSTM1, GSTP1, GSTT1, CYP1A1, CYP1A2, CYP1B1, CYP2A6, CYP2B6, CYP2C9, CYP1C19, CYP2D6, CYP2E1, CYP3A4, and $C Y P 3 A 5$ genes through Multiplex Ligation-Dependent Probe Amplification (MLPA) in 123 healthy individuals from a cohort belonging to the Colombian population. Our results indicated that 13 out of 14 genes exhibited CNVs defined by the presence of deletions and/or duplications in at least one exon. 33.3\% of the genes presented the combination of both. Our population exhibited variability in CNVs: $50 \%$ of individuals carried deletions and duplications while 39\% had a unique type of CNV (deletion or duplication). According to the number of CYP-450 or GST active copies, the individuals can be potentially defined as poor metabolizers (PM) or ultrarapid metabolizers (UM) [14]. We identified that $83 \%$ of the analyzed individuals presented CNVs in one or several of the CYP-450 and / or GST genes studied.

Our results constitute the first description of the frequency of CNVs in a Colombian cohort, contributing to the knowledge of these CNVs in the Latin American population and their potential utilization in the clinical setting.

\section{Methods}

\section{Study population}

Peripheral blood samples were obtained for DNA extraction from 123 healthy donors from the Center For Research in
Genetics and Genomics (Bogotá, Colombia). More precisely, detailed information on the methodology for healthy individuals' enrollment was included in the internet site of the institution. Each participant was informed with respect to: project objectives, sampling procedure, risks and results management. All the individuals signed an informed consent regarding the use of their DNA for research. $58 \%$ of the participants were women and $42 \%$ men, with ages ranging from 20 to 59 years. All the chosen subjects were born in Bogota, the capital of Colombia, a city with an estimated population structure with a predominance of native ancestry (52\%), followed by European and African (45 and 3\% respectively). None of the participants were asked for their self-reported ethnicity, and ancestry was assumed as indicated in previous studies based on the analysis of AIM's in individuals from this same population. [15] . The sample size was calculated considering the estimation of a proportion with a confidence level of $95 \%$ ( $\alpha: 0.05$, z: 1.96), p (sample proportion) 3\% and e (margin of error) 3\% [16]. Considering that this is the first study that analyzes genomic regions in 14 CYP-450 and GST genes by MLPA in the Colombian population, the value of sample proportion (p) was estimated according to the frequency of alleles with duplication/deletion of the CYP2D6 gene identified by Isaza et al. [17]. The sample size (with finite population correction and) was equal to 125 .

The experimental procedures of this study were approved by the Ethics Committee of Universidad del Rosario (CEIAMH002-000174). The study was conducted according to the principles of the Helsinki Declaration (institutional review board reference CS/ABN062).

\section{Multiplex ligation-dependent probe amplification (MLPA)} Genomic DNA was isolated from blood samples using the Salting-out method. MLPA was performed using the commercial kit SALSA MLPA P128-C1 Cytochrome P450 probe mix (\#P128-C1, MRC-Holland, Amsterdam) according to the manufacturer's instructions. As stated by the information from the kit, the P128-C1 Cytochrome P450 probemix contains 52 MLPA probes with amplified products between 128 and 504 nt. Additionally it includes 4 DNA quantity fragments (Q-fragments), three DNA denaturation control (D.-fragments), an Xfragment and one Y-fragment (https://www.mlpa.com). For the identification of the CNVs, 40 genomic regions in 14 CYP-450 and GST genes, which were contained in the commercial kit, were used in this analysis (Table 1).

Each gene was analyzed with at least two probes, with the exception of GSTT1, which was determined by one probe in the exon 1 . The genes of the cytochrome P450 and Glutathione S-transferase included in the analysis were: GSTM1, GSTP1, GSTT1, CYP1A1, CYP1A2, CYP1B1, CYP2A6, CYP2B6, CYP2C9, CYP2C19, CYP2D6, CYP2E1, CYP3A4, and CYP3A5. 
Table 1 Genomic Regions analyzed

\begin{tabular}{|c|c|c|c|c|c|c|c|c|c|c|c|c|}
\hline & Exon1 & Exon2 & Exon3 & Exon4 & Exon5 & Exon6 & Exon7 & Exon8 & Exon9 & Exon10 & Exon13 & Downstream Exon9 \\
\hline$\overline{C Y P 1 A 1}$ & $\checkmark$ & 2 & $\sqrt{2}$ & & & & & & & & & \\
\hline CYPIAZ & & $\checkmark$ & & $\checkmark$ & & & $\checkmark$ & & & & & \\
\hline CYPIB1 & $\checkmark$ & & $\checkmark$ & & & & & & & & & \\
\hline CYP2A6 & $\checkmark$ & $\checkmark$ & $\checkmark$ & & $\checkmark$ & & & & & & & \\
\hline CYP2B6 & & $\checkmark$ & $\checkmark$ & $\checkmark$ & & & & & & & & \\
\hline CYP2C19 & & $\checkmark$ & & & & $\checkmark$ & & & $\checkmark$ & & & \\
\hline CYP2C9 & $\checkmark$ & & & & & & $\checkmark$ & $\checkmark$ & $\checkmark$ & & & \\
\hline CYP2D6 & $\checkmark$ & & & & $\checkmark$ & $\checkmark$ & & & & & & $\checkmark$ \\
\hline CYP2E1 & & & & & $\checkmark$ & $\checkmark$ & & $\checkmark$ & & & & \\
\hline СYPЗА4 & $\checkmark$ & & & & & $\checkmark$ & & & & & $\checkmark$ & \\
\hline CYP3A5 & & $\checkmark$ & & $\checkmark$ & & & & & & $\checkmark$ & & \\
\hline GSTM1 & & & $\checkmark$ & & $\checkmark$ & & & & & & & \\
\hline GSTP1 & & & $\checkmark$ & $\checkmark$ & & & & & & & & \\
\hline GSTT1 & $\checkmark$ & & & & & & & & & & & \\
\hline
\end{tabular}

For each MLPA reaction, $50 \mathrm{ng}$ of DNA of each sample was denatured in a thermocycler for $5 \mathrm{~min}$ at $98^{\circ} \mathrm{C}$. After cooling to $25^{\circ} \mathrm{C}$, the probemix and the MLPA buffer were added to each sample, mixed and incubated for $1 \mathrm{~min}$ at $95^{\circ} \mathrm{C}$ followed by $16 \mathrm{~h}$ of hybridization at $60^{\circ} \mathrm{C}$. The ligation reaction was performed incubating at $54{ }^{\circ} \mathrm{C}$ the ligase- $65 \mathrm{mix}$, followed by heating at $98^{\circ} \mathrm{C}$ for $5 \mathrm{~min}$. Thereafter PCR was performed using exon-specific probes with universal-tagged primers. The PCR consisted of 35 amplification cycles, $\left(95^{\circ} \mathrm{C}\right.$ for $30 \mathrm{~s}, 60^{\circ} \mathrm{C}$ for $30 \mathrm{~s}$ and $72^{\circ} \mathrm{C}$ for $1 \mathrm{~min}$ ), followed by a 20 -min incubation at $72^{\circ} \mathrm{C}$. The amplified products were separated by capillary gel electrophoresis in an Applied Biosystems 3500 Genetic Analyzer using GeneScan350 ROX as standard internal lane size.

\section{Data analysis}

The analysis of MLPA was performed using coffalyser.Net software (https://www.mlpa.com). Data generated by SALSA MLPA P128-C1 Cytochrome P450 probe mix was normalized intra-sample (within each sample, compare each probe peak to the peaks of the reference probes). The relative probe signals determined are then used in intersample normalisation (final probe ratios are determined by comparing the relative probe peak in the DNA sample of interest to all reference samples.). The quality control and data normalization were performed using reference probes (SALSA MLPA P128-C1 Cytochrome $\mathrm{P} 450$ probe mix).

CNV status was assigned as follows: if a deletion or duplication on either of the exons in the gene was detected, the whole gene was categorized as deleted or duplicated respectively. The copy number was determined in accordance with instructions of SALSA MLPA
P128-C1 Cytochrome P450 probe mix. The relationship between copy number status and the typical distribution of Dosage Quotient Distribution (DQs) (based on a large number of samples at MRC-Holland) was: $D Q=0$ (homozygous deletion); $0.40<\mathrm{DQ}<0.65$ (heterozygous deletion); $0.80<\mathrm{DQ}<1.20$ (Normal); $1.30<\mathrm{DQ}<1.65$ (heterozygous duplication); $1.75<\mathrm{DQ}<2.15$ (homozygous duplication), all other values (ambiguous result) (www.mlpa.com).

All the samples were divided into 14 categories based on the genotype combination of the 14 genes. Moreover, we determined the frequency of the individuals for each category. Likewise, individuals were categorized into 4 defined groups: if they had only deletions in one or more genes, only duplications, deletions and duplications or no CNVs. The analysis for the allelic and genotypic frequencies for each gene was determined using SNPStats (https://www.SNPstats.net/start.htm).

\section{Results}

CNVs were analyzed using a panel of MLPA with 11 genes from the family of cytochrome P-450 (CYP1A1, CYP1A2, CYP1B1, CYP2A6, CYP2B6, CYP2C9, CYP2C19, CYP2D6, CYP2E1, CYP3A4, CYP3A5) and 3 from the glutathione S- Transferase family (GSTM1, GSTP1, GSTT1). In $92.9 \%$ of the genes, there were duplications and/or deletions identified, CYP1A2 was the only gene with no CNVs identified. The frequency of deletions and duplications were 0 to $50.4 \%$ and 0 to $18.7 \%$ respectively (Fig. 1). Our study revealed that CNVs were frequent in the glutathione S-transferase genes, GSTM1 showed a percentage of individuals with deletion-duplication of $67 \%$, followed by GSST1 with $54 \%$. In CYP-450 genes, CYP2D6 was the most polymorphic (13\% duplication 


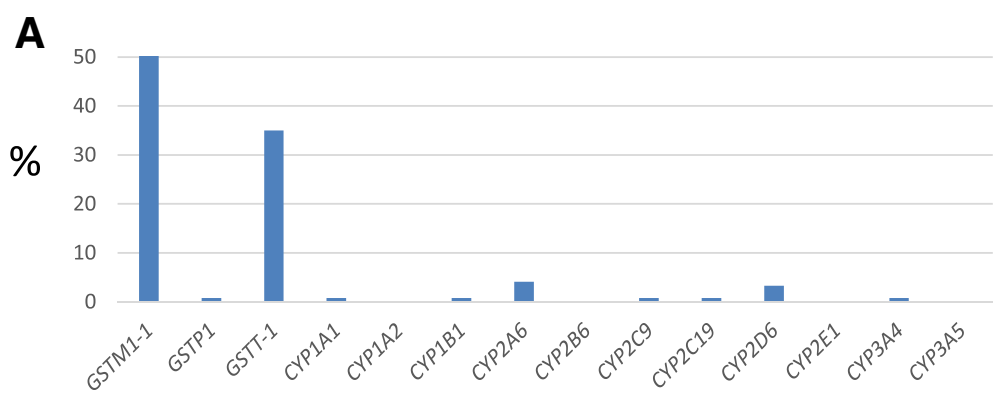

B
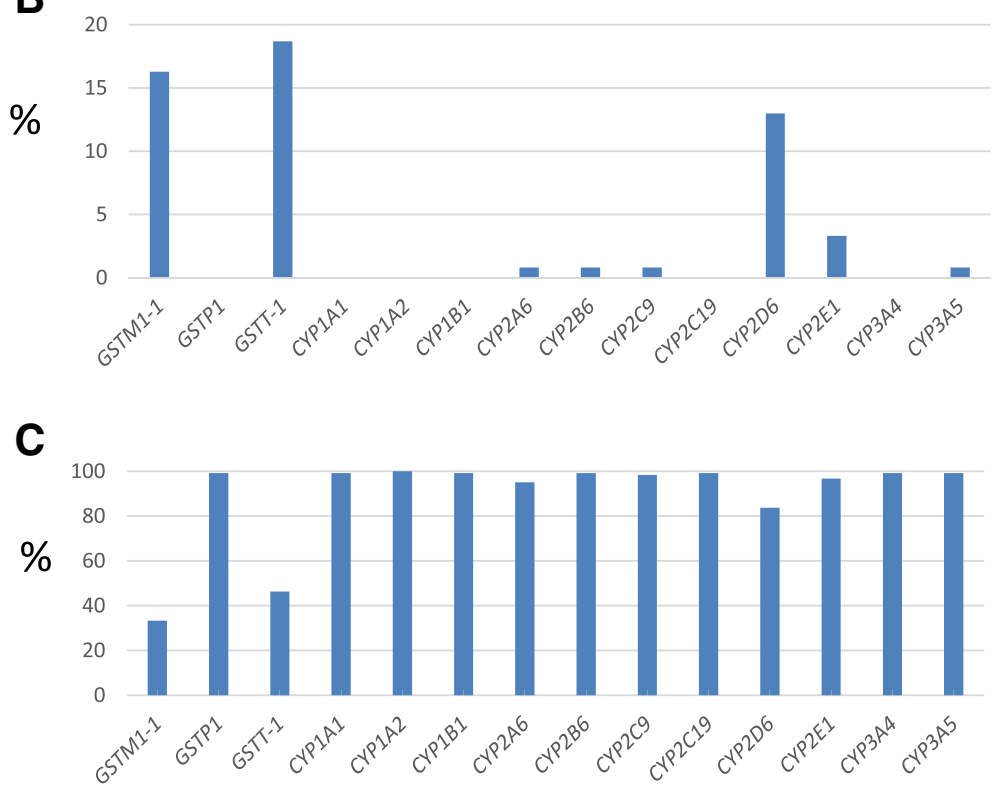

Fig. 1 Copy number variation in CYP-450 and GST genes. a Deletions, b Duplications, c No CNVs

and $3.3 \%$ deletion). Thirteen out of 14 genes have some CNVs: 5 (35.7\%) exhibited deletions and duplications while and 8 (57.1\%) only deletions or duplications.

The samples were distributed into 14 categories based on the mutational status of the 14 genes analyzed: 1) wild-type for all genes; 2) homozygous deletion for one or more genes; 3) heterozygous deletion for one or more genes; 4) homo or heterozygous deletion for one or more genes; 5) homozygous duplication for one or more genes; 6) heterozygous duplication for one or more genes; 7) hetero and homozygous deletions; 8) heterozygous duplications/homozygous deletions; 9) homozygous duplications/heterozygous deletions; 10) homozygous duplications/ homozygous deletions; 11) homozygous duplications/heterozygous duplications; 12) heterozygous duplications/homo y heterozygous deletions; 13) hetero and homozygous duplications/heterozygous deletions and 14) hetero and homozygous duplications/homozygous deletions (Table 2)In categories 1, 2 and 10 we observed the greatest number of individuals $(17,23$, and $12 \%$ respectively). $35 \%$ of subjects were carries of different combinations of $\mathrm{CNVs}$ (category 7-14). When categorizing the individuals according to the type of $\mathrm{CNV}$, it was possible to establish that most of them were carriers of only deletions (38\%), while the presentation of exclusive duplications was only evidenced in $11 \%$ of the population. The combination of CNVs was identified in $33 \%$ of the cases and no CNV were identified in $17 \%$ (Fig. 2).

The genotypic frequencies established for each gene are described in Table 3. The allelic frequencies of the CYP and GST studied genes were determined with ranges for alleles deleted from 0 to $47.6 \%$, alleles duplicated from 0 to $17.5 \%$ and normal alleles from 37 to $100 \%$ (Table 4 ).

\section{Discussion}

The analysis of genomic variation in the general population is essential to understand the phenotypic diversity and its potential involvement in drug response. The Human Genome Project $[11,18]$, the SNP Consortium, The International Hap Map project [19], and more recently the 1000 Genomes Project and the Encyclopedia of DNA Elements (ENCODE) have collectively identified nearly 12 million 
Table 2 Population categorization by mutational status

\begin{tabular}{|c|c|c|}
\hline Category & Mutational Status & $\begin{array}{l}\text { Percentage } \\
(\%)\end{array}$ \\
\hline 1. & Wild type & 17 \\
\hline 2. & Homozygous deletions & 23 \\
\hline 3. & Heterozygous deletions & 7 \\
\hline 4. & Homo and heterozygous deletions & 9 \\
\hline 5. & Homozygous duplications & 9 \\
\hline 6. & Heterozygous duplications & 1 \\
\hline 7. & Hetero and homozygous deletions & 2 \\
\hline 8. & $\begin{array}{l}\text { Heterozygous duplications and homozygous } \\
\text { deletions }\end{array}$ & 10 \\
\hline 9. & $\begin{array}{l}\text { Homozygous duplications and heterozygous } \\
\text { deletions }\end{array}$ & 5 \\
\hline 10. & $\begin{array}{l}\text { Homozygous duplications and homozygous } \\
\text { deletions }\end{array}$ & 12 \\
\hline 11. & $\begin{array}{l}\text { Homozygous duplications and heterozygous } \\
\text { duplications }\end{array}$ & 2 \\
\hline 12. & $\begin{array}{l}\text { Heterozygous duplications, homozygous and } \\
\text { heterozygous deletions }\end{array}$ & 2 \\
\hline 13. & $\begin{array}{l}\text { Heterozygous deletions, Heterozygous and } \\
\text { homozygous duplications }\end{array}$ & 2 \\
\hline 14. & $\begin{array}{l}\text { Hetero and homozygous duplications and } \\
\text { homozygous deletions }\end{array}$ & 1 \\
\hline
\end{tabular}

SNP representing 26 populations around the world $[20,21]$. Less is known on CNVs, even though they are suspected to be involved in genetic disease susceptibility and the efficacy/toxicity response to drugs $[1,9,12,22]$. Due to current knowledge of CNVs in relation to drug efficacy and toxicity and the fact that its variation in Latin Americans is understudied, it's necessary to conduct studies in these CNVs. In our study, the presence of CNVs were evaluated using the commercial kit SALSA MLPA P128-C1 Cytochrome P450 probe mix (\#P128-C1, MRC-Holland, Amsterdam). At present, the SALSA MLPA
P128-C1 Cytochrome P450 probe mix is the only available commercial analytical panel design for analyzing Cytochromes P450 (CYP) genes deletions and duplications (https://www.mlpa.com) [23-25]. Genomic variants in human CYPs are a major source of variability in drug pharmacokinetics and response. CYP1, 2, and 3 families, are the principal metabolizing enzymes of phase I metabolism involved in most of medications [26-28]. Additionally, the panel includes GST genes related to detoxification of carcinogens, therapeutic chemicals and environmental toxins [29]. The accurate understanding of genomic variants prevalence related to drug toxicity and efficacy is important for proposing adequate therapeutic management.

We observed that 13 out of 14 genes studied (92.9\%) presented deletions and/or duplications. Regarding these findings, the analysis of CNVs in 542 healthy unrelated individuals showed polymorphisms in 3 out of 11 CYP450 genes [6], while another study of CNVs found that $C Y P 1 A 1, C Y P 1 B 1$, and CYP2B6 had no CNVs [29]. We consider that in the Colombian population there is greater variability in the GST and CYP-450 genes than in other populations. Testing CNVs in GSTs and CYPs genes can allow the selection of patients for different starting dose regimes; indeed $\mathrm{CNVs}$ genotypes of these genes are predictors of response to treatment [24].

Our results indicated that nearly $50 \%$ of our population had one type of CNV (deletion or duplication) in one or several of the analyzed genes and 33\% of the individuals had some genes with deletion and others with duplication. The individuals carrying nonactive alleles are classified as "predicted" poor metabolizers with no metabolic capacity for these enzymes. On the contrary, individuals with more than two active alleles have been associated with increased enzyme activity [14]. There are two risk scenarios in regards to the genetic background: the first is the administration of a prodrug that requires conversion to an active metabolite where slow metabolizers generate loss of

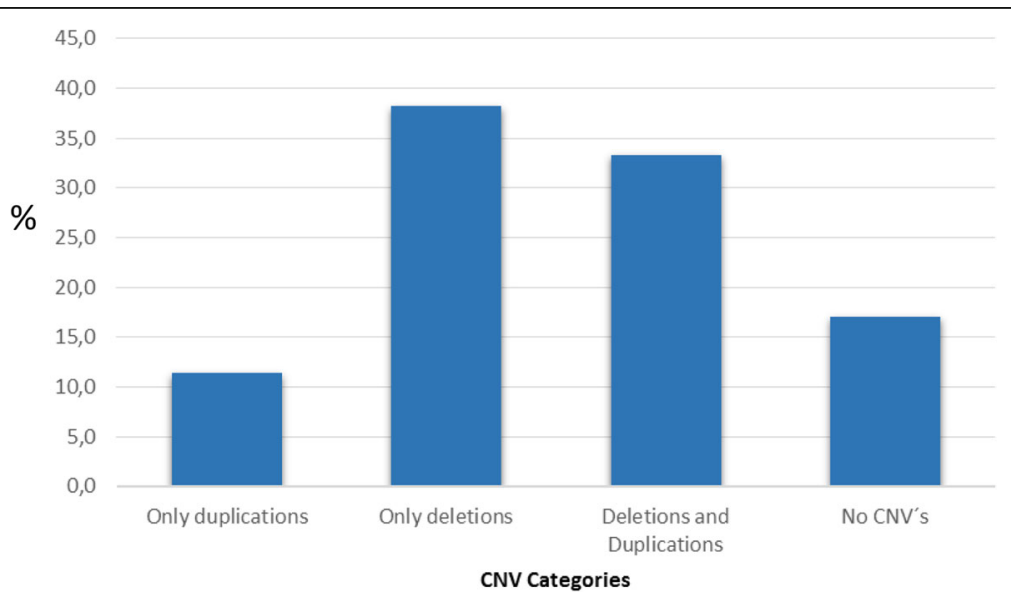

Fig. 2 Distribution of CNVs in analyzed genes 
Table 3 Genotypic frequencies

\begin{tabular}{lllllllllllllll}
\hline & GSTM1 & GSTP1 & GSTT1 & CYP1A1 & CYP1A2 & CYP1B1 & CYP2A6 & CYP2B6 & CYP2C9 & CY2C19 & CYP2D6 & CYP2E1 & CYP3A4 & CYP3A5 \\
\hline Del/Wt & 5.7 & 0.8 & 15.4 & 0.8 & 0 & 0.8 & 4.1 & 0 & 0.8 & 0.8 & 3.3 & 0 & 0.8 & 0 \\
Del/Del & 44.7 & 0 & 19.5 & 0 & 0 & 0 & 0 & 0 & 0 & 0 & 0 & 0 & 0 & 0 \\
Dup/Wt & 1.6 & 0 & 2.4 & 0 & 0 & 0 & 0.8 & 0.8 & 0.8 & 0 & 10.6 & 3.3 & 0 & 0.8 \\
Dup/Dup & 14.6 & 0 & 16.3 & 0 & 0 & 0 & 0 & 0 & 0 & 0 & 2.4 & 0 & 0 & 0 \\
Wt/Wt & 33.3 & 99.2 & 46.3 & 99.2 & 100 & 99.2 & 95.1 & 99.2 & 98.4 & 99.2 & 83.7 & 96.7 & 99.2 & 99.2 \\
\hline
\end{tabular}

efficacy. The second is the administration of a drug that is eliminated by a single pathway since its absence results in the accumulation of the parent drug generating toxicity. Coadministration of drugs that inhibit a bioactivating enzyme can result in "phenocopy" of the slow metabolizer phenotype [30]. Our study has revealed the existence of genotypic diversity that allowed the identification of 14 categories defined by the mutational status observed in the 14 genes included. $35 \%$ of the individuals were carriers of different combinations of CNVs that reflect the dynamics underlying this type of variant. Several mechanisms have been proposed in the generation of a CNV including nonallele homologous recombination, non-homologous end joining, fork stalling and template switching, and microhomology-mediated break-induced replication.

To date, findings show that $\mathrm{CNV}$ duplications are significantly more frequent than deletions [5, 31]; however, our study identified deletions as the predominant mutation. The allelic frequencies of deletions were greater in the GST than in the CYP-450 genes: for GSTM-1 the biggest value in the population was identified (50.4\%) followed by GSST-1 (35\%), while for CYP the highest deletion allelic frequencies reported were 3.3\% (CYP2D6) and 4.1\% (CYP2A6). Regarding GST genes, their location among genomic regions of segmental duplications (SD) is relevant, since the regions flanked by SD are prone to rearrangement by nonallelic homologous recombination [32-34]. Our results are in concordance with others and indicate that deletions in GST genes are relatively common in different populations $(23,7 \%$ to $51,6 \%$ for GSTM1 and $4,25 \%$ to $46,8 \%$ for GSTT1) [12]. According to the function of GSST1 and GSTM1 in the detoxification of exogenous compounds, the individuals carrying deletions have an increased risk for several cancers (colorectal and chronic myeloid leukemia) and toxicities related to medications [35-39]. Some of the toxicity reactions are secondary to a combination of deletions in GSST1 and GSTM1 genes. Given the high prevalence of deletions in GSST1 and GSTM1, $16.2 \%$ of the participants in our study were carriers of these double mutations, a finding that led to estimate the potential impact of these variants in our cohort of Colombian individuals.

The presence of deletions in other genes (CYP-450 and GSTP1) was lower (0 to $1.6 \%$ ) in relation to GSST1 and GSTM1. Moreover, CYP2D6 and CYP2A6 presented an allele frequency greater than $1 \%$ with values of 1.6 and $2.0 \%$ respectively. With the exception of CYP2D6, little is known about the frequency of CNVs in these genes. The clinical and pharmacogenomic implication of CYP2A6 deletion has been related to its role in the metabolism of nicotine, cotinine and nitrosamine, precarcinogens which increase the risk of tobacco-related cancer [40, 41]. In our study, allelic and genotypic frequencies for CYP1A1 and CYP1B1 null were 0.4 and $0.8 \%$ respectively. The genetic population characteristics of these CNVs are unknown. It has been estimated that the pharmacogenetic impact of CYP1A1 and CYP1B1 is lower compared to other family members of CYP-450, due to the fact that they are extrahepatic enzymes, and therefore have limited relevance in the elimination of substrates (caffeine, phenacetin, flunarizine, amiodarone and others). In accordance to other reports, our findings demonstrate an absence of CNVs in CYP1A2 [42], suggesting that CYP1A2 is a conserved gene, for which common variants that significantly alter gene expression or enzyme activity have not been described [33]. Regarding the members of the CYP2 family, allelic frequencies for $C Y P 2 C 9$ and $C Y P 2 C 19$ were identical $(0.4 \%)$. Our findings, which are similar to those proposed by other authors, indicate that CYP2C9 and CYP2C19 duplications/deletions are rare in the population [43]. These findings suggest that the influence of CNVs in CYP2C9 and CYP2C19 in the pharmacological responses is less significant than SNVs.

Table 4 Allelic frequencies

\begin{tabular}{lllllllllllllll}
\hline & GSTM1 & GSTP1 & GSTT1 & CYP1A1 & CYP1A2 & CYP1B1 & CYP2A6 & CYP2B6 & CYP2C9 & CY2C19 & CYP2D6 & CYP2E1 & CYP3A4 & CYP3A5 \\
\hline Deletion & 47.6 & 0.4 & 27.2 & 0.4 & 0 & 0.4 & 2.0 & 0 & 0.4 & 0.4 & 1.6 & 0 & 0.4 & 0 \\
Duplication & 15.4 & 0 & 17.5 & 0 & 0 & 0 & 0.4 & 0.4 & 0.4 & 0 & 7.7 & 1.6 & 0 & 0.4 \\
No CNV's & 37.0 & 99.6 & 55.3 & 99.6 & 100 & 99.6 & 97.6 & 99.6 & 99.2 & 99.6 & 90.7 & 98.4 & 99.6 & 99.6 \\
\hline
\end{tabular}


CYP2D6 metabolizes over $25 \%$ of drugs currently used in the clinical practice [26]. The deletion of the whole gene was present in our population with an allelic frequency of $1.6 \%$ in accordance with other admixed Americans populations (3\%). Worldwide, there is ethnic variability with frequencies from 2 to $6.5 \%$, which contribute essentially to the interindividual variability in the response to drugs observed in different populations [44]. The medical response of the CYP2D6 deletion carriers has been widely documented and associated with the occurrence of ADRs generated by the high levels of parenteral drugs or by therapeutic failure secondary to the inability to create an active metabolite [45]. The duplications and multiduplications have been associated to CYP2D6, with individuals that carry among 2 to 13 gene copies. Our results indicated that the genotypic frequency of CYP2D6WT/CYP2D6Dup was $10.6 \%$, while homozygotes for the polymorphism corresponded to $2.4 \%$. The allelic frequency for duplications was $7.7 \%$, greater than the reported by Zhou et al. (1\%), who studied 5789 samples of admixed Americans [44]. It is possible that our population has its own profile in genes such as CYP2D6. Individuals with extra copies of CYP2D6 correspond to the UM group in which each functional copy increases the metabolism rate of the enzyme substrate. The relationship between genotype and phenotype should be analyzed with caution since, although it has been established that heritability of interindividual differences of the drug response phenotype is near to $70 \%$, the analysis of common variants has explained less than half of estimate heritability. Rare variants, different types of genomic variation and factors such as drug-drug interactions, are determinants in the multifactorial or complex behavior of the metabolic phenotype [46]. Recently, the emergence of "pharmacometabolomic-aided pharmacogenomics" reinforces the need to clinically identify and validate the potential associations of genetic, physiological, chemical and environmental influence related to the toxicity / efficacy of xenobiotics. This synergy can have a great impact in predicting the benefit of the therapeutic intervention in patients [47]. Some reports have established the need to analyze the clinical implication of pharmacogenetics from the genotypic, haplotype and phenotypic perspective and not only focus on one level of information, since genomic variants can vary throughout the different populations and their effect on the phenotype of interest can be modified by one or more variants [48]. For Latin American populations, including Colombia, it is common to see mixed populations with different percentages of ancestries (Table 5) and it is recognized that it is a continuous rather than a categorical variable, even within the self-reported race / color categories [49].

Our results highlight the variability and potential impact of GST and CYP-450 genes in interindividual drug response. In terms of pharmacogenetic evaluation, we estimate that our results indicate that in the Colombian population there exists a significant allele frequency conferring susceptibility for an inadequate response to certain drugs; GSTM1, GSTT1, CYP2D6, and $C Y P 2 A 6$ showed the greatest variability of CNVs. Duplications and deletions in CYP2D6 $(9.3 \%$ of the alleles identified) influence drug pharmacokinetics and subsequent pharmacological and toxicological effects

Table 5 Ancestry in different regions of Colombia [15]

\begin{tabular}{|c|c|c|c|c|c|}
\hline & & Type Marker & European (\%) & African(\%) & Amerindian(\%) \\
\hline \multirow[t]{2}{*}{ Caribbean area } & Santa Marta (n:26) & AlMs & 50 & 28 & 22 \\
\hline & Cartagena (n:80) & AIMs & 23 & 44 & 33 \\
\hline \multirow[t]{4}{*}{ Northwest } & Medellin (n:849) & AIMs & 60 & 12 & 28 \\
\hline & Peque (n:163) & AIMs & 32 & 6 & 62 \\
\hline & Manizales(n:203) & AIMs & 59 & 4 & 37 \\
\hline & Bucaramanga(n:82) & AIMs & 56 & 1 & 43 \\
\hline \multirow[t]{5}{*}{ Central } & Armenia(n:58) & AIMs & 57 & 5 & 38 \\
\hline & Bogotá(n:24) & AIMs & 45 & 3 & 52 \\
\hline & Boyacá(n:80) & SNPS & 42 & 20 & 38 \\
\hline & Cundinamarca(n:19) & STRs & 47 & 2 & 51 \\
\hline & Huila(n:82) & SNPS & 41 & 19 & 40 \\
\hline \multirow[t]{3}{*}{ Southwest } & Pasto(n:201) & AIMs & 32 & 3 & 65 \\
\hline & Popayán(n:61) & AIMs & 20 & 23 & 57 \\
\hline & Neiva(n:24) & AlMs & 39 & 0 & 61 \\
\hline Pacific coast & Quibdo(n:72) & AIMs & 21 & 68 & 11 \\
\hline
\end{tabular}


[7].Those genomic variants impact about $25 \%$ of the drugs used clinically (e.g. amiodarone, amitriptyline, clomipramine, codeine, tramadol, fluoxetine, simvastatin) in therapeutic areas related to psychiatry, cardiology, and oncology [28]. The analysis of CNVs for CYP2D6 has been documented in clinical management guidelines established by international consortiums such as the Dutch Pharmacogenetics Working Group guidelines (DPWG), and the Clinical Pharmacogenetics Implementation Consortium (CPIC). Clinical evidence has suggested that CYP2D6 genetic testing provide useful information to guide drug dosage and interpretation of potential patients' metabolizer phenotypes. Concerning the GST genes, the individuals carrying deleted alleles (e.g. GSTT1 and GSTM1 null) are of special interest regarding the response to antineoplastic agents for cancer treatment. Interestingly, since CYP2A6 variants have been related to antiretroviral therapy our results might be useful for delineate therapeutic strategy accurately in Colombian HIV/ AIDS patients. Until now, although some important evidence regarding the impact of CNVs on the toxicity and efficacy of drug response has been published, the translation of this knowledge into clinical practice has not been widely determined. The incorporation of CNVs genetic testing into health system is therefore still uncertain.

Taking together, our results allow us to establish for the first time a profile of CNVs for GST and CYP-450 genes in a cohort of Colombian individuals. We estimate that our results are representative for the Colombian and Latin American population with ancestry (reported in the literature by AIMs) similar to that attributed to the healthy people evaluated in this work (Table 5).

We consider that the principal limitation is the nondetection of copy number changes that lie outside the target sequences of probes incorporated in the SALPA MLPA P128-C1 Cytochrome $\mathrm{P} 450$ probe mix. In the case of CYP2D6, MLPA does not allow the discrimination of the presence of duplications in active genes, which would require additional analysis capable of identifying CNVs and SNVs simultaneously. Additionally, our study lacks an ancestry analysis of the participants; therefore genetic background cannot be established with accurately.

\section{Conclusion}

Our results describe the first genomic profile of CNVs for GST and CYP genes in a cohort of the Colombian population. These findings are relevant due to the impact of these genes in the pharmacogenomic drug selection and dosing, adverse drug reactions and disease susceptibility. Additionally, our search serves to the understanding of the CNVs frequency and potential health impact, so far unknown in other Latin American populations.

\section{Abbreviations}

ADME: Drug absorption, distribution, metabolism and excretion;

ADRs: Adverse drug reactions; AIMs: Ancestry informative marker sets; CNVs: Copy number variations; EMA: The European Medicines Agency; ENCODE: The Encyclopedia of DNA Elements; FDA: US Food and Drug Administration; MLPA: Multiplex ligation-dependent probe amplification; PCR: Polymerase chain reaction; PM: Poor metabolizer; SD: Segmental duplications; SNVs: Simple nucleotide variants; UM: Ultrarapid metabolizers

\section{Acknowledgements}

We would like to thank Dr. Daniela Duarte and Dr. Luz Miriam Siza for her help during MLPAexperiments.

\section{Authors' contributions}

$\mathrm{BR}, \mathrm{MJN}, \mathrm{DC}, \mathrm{KA}, \mathrm{KQ}, \mathrm{NC}, \mathrm{CTA}$, and MAG, participated in collecting the samples, MLPA and Data analysis; PL performed results analysis and helped to draft the manuscript; DJF designed, directed the study, and drafted the paper. All authors revised and approved the last version of the manuscript.

\section{Funding}

This project was supported by the Universidad del Rosario (Grant CS/ ABN062). The fund was mainly used for DNA extraction and MLPA analysis (commercial kit SALSA MLPA P128-C1 Cytochrome P450 probe mix (\#P128C1, MRC-Holland, Amsterdam)

\section{Availability of data and materials}

The software of MLPA analysis used during the current study is available at https://www.mlpa.com/WebForms/WebFormMain.aspx. Data obtained in our study is available from the corresponding author on request.

\section{Ethics approval and consent to participate}

All the individuals signed an informed consent regarding the use of their DNA for research. All this study's experimental steps were approved by the Universidad del Rosario's Ethics Committee (IRB-CEI-AMH002-000174); the study was conducted in line with the Declaration of Helsinki (approval date: June 24 2008)

\section{Consent for publication}

Not applicable

\section{Competing interests}

The authors declare that they have no competing interests.

Received: 8 May 2019 Accepted: 5 July 2019

Published online: 19 July 2019

\section{References}

1. Santos M, Niemi M, Hiratsuka M, Kumondai M, Ingelman-Sundberg M, Lauschke VM, Rodriguez-Antona C. Novel copy-number variations in pharmacogenes contribute to interindividual differences in drug pharmacokinetics. Genet Med. 2018;20(6):622-9.

2. Li J, Zhang L, Zhou H, Stoneking M, Tang K. Global patterns of genetic diversity and signals of natural selection for human ADME genes. Hum Mol Genet. 2011;20(3):528-40.

3. Sim SC, Kacevska M, Ingelman-Sundberg M. Pharmacogenomics of drugmetabolizing enzymes: a recent update on clinical implications and endogenous effects. Pharmacogenomics J. 2013;13(1):1-11.

4. Johansson I, Ingelman-Sundberg M. CNVs of human genes and their implication in pharmacogenetics. Cytogenet Genome Res. 2008;123(1-4):195-204.

5. Redon R, Ishikawa S, Fitch KR, Feuk L, Perry GH, Andrews TD, Fiegler H, Shapero MH, Carson AR, Chen W, et al. Global variation in copy number in the human genome. Nature. 2006;444(7118):444-54.

6. Martis S, Mei H, Vijzelaar R, Edelmann L, Desnick RJ, Scott SA. Multi-ethnic cytochrome-P450 copy number profiling: novel pharmacogenetic alleles and mechanism of copy number variation formation. Pharmacogenomics J. 2013;13(6):558-66.

7. Ouahchi K, Lindeman N, Lee C. Copy number variants and pharmacogenomics. Pharmacogenomics. 2006;7(1):25-9.

8. Nakamura T, Ohnuma T, Hanzawa R, Takebayashi Y, Takeda M, Nishimon S, Sannohe T, Katsuta N, Higashiyama R, Shibata N, et al. Associations of common copy number variants in glutathione S-transferase mu 1 and D-dopachrome 
tautomerase-like protein genes with risk of schizophrenia in a Japanese population. Am J Med Genet B Neuropsychiatr Genet. 2015;168(7):630-6.

9. Kim IW, Han N, Kim MG, Kim T, Oh JM. Copy number variability analysis of pharmacogenes in patients with lymphoma, leukemia, hepatocellular, and lung carcinoma using the Cancer genome atlas data. Pharmacogenet Genomics. 2015;25(1):1-7.

10. Naranjo MG, Rodrigues-Soares F, Penas-Lledo EM, Tarazona-Santos E, Farinas H, Rodeiro I, Teran E, Grazina M, Moya GE, Lopez-Lopez M, et al. Interethnic variability in CYP2D6, CYP2C9, and CYP2C19 genes and predicted drug metabolism phenotypes among 6060 Ibero- and native Americans: RIBEF-CEIBA consortium report on population pharmacogenomics. Omics. 2018;22(9):575-88.

11. Adhikari K, Mendoza-Revilla J, Chacon-Duque JC, Fuentes-Guajardo M, RuizLinares A. Admixture in Latin America. Curr Opin Genet Dev. 2016;41:106-14.

12. He Y, Hoskins JM, McLeod HL. Copy number variants in pharmacogenetic genes. Trends Mol Med. 2011;17(5):244-51.

13. Ihnatovych I, Nayak TK, Ouf A, Sule N, Birkaya B, Chaves L, Auerbach A, Szigeti K. iPSC model of CHRFAM7A effect on alpha7 nicotinic acetylcholine receptor function in the human context. Transl Psychiatry. 2019;9(1):59.

14. Tanoshima R, Khan A, Biala AK, Trueman JN, Drogemoller BI, Wright GEB, Hasbullah JS, Groeneweg GSS, Ross CDD, Carleton BC. Analyses of adverse drug reactions-Nationwide active surveillance network: Canadian pharmacogenomics network for drug safety database. J Clin Pharmacol. 2019;59:356-63.

15. Salzano FM, Sans M. Interethnic admixture and the evolution of Latin American populations. Genet Mol Biol. 2014;37(1 Suppl):151-70.

16. Kadam P, Bhalerao S. Sample size calculation. Int J Ayurveda Res. 2010;1(1):55-7.

17. Isaza CA, Henao J, Lopez AM, Cacabelos R. Isolation, sequence and genotyping of the drug metabolizer CYP2D6 gene in the Colombian population. Methods Find Exp Clin Pharmacol. 2000;22(9):695-705.

18. Venter JC, Smith HO, Adams MD. The sequence of the human genome. Clin Chem. 2015;61(9):1207-8.

19. International HapMap Consortium. A haplotype map of the human genome. Nature. 2005:437(7063):1299-320.

20. Manry J, Quintana-Murci L. A genome-wide perspective of human diversity and its implications in infectious disease. Cold Spring Harb Perspect Med. 2013;3(1):a012450.

21. Farris MH, Scott AR, Texter PA, Bartlett M, Coleman P, Masters D. TIA: algorithms for development of identity-linked SNP islands for analysis by massively parallel DNA sequencing. BMC Bioinformatics. 2018;19(1):126.

22. Mikhail FM. Copy number variations and human genetic disease. Curr Opin Pediatr. 2014;26(6):646-52.

23. Stuppia L, Antonucci I, Palka G, Gatta V. Use of the MLPA assay in the molecular diagnosis of gene copy number alterations in human genetic diseases. Int J Mol Sci. 2012;13(3):3245-76.

24. Shen Y, Wu BL. Designing a simple multiplex ligation-dependent probe amplification (MLPA) assay for rapid detection of copy number variants in the genome. J Genet Genomics. 2009;36(4):257-65.

25. Sellner LN, Taylor GR. MLPA and MAPH: new techniques for detection of gene deletions. Hum Mutat. 2004;23(5):413-9.

26. Zhou SF, Liu JP, Chowbay B. Polymorphism of human cytochrome P450 enzymes and its clinical impact. Drug Metab Rev. 2009;41(2):89-295.

27. Elfaki I, Mir R, Almutairi FM, Duhier FMA. Cytochrome P450: polymorphisms and roles in Cancer, diabetes and atherosclerosis. Asian Pac J Cancer Prev. 2018;19(8):2057-70.

28. Tornio A, Backman JT. Cytochrome P450 in Pharmacogenetics: an update. Adv Pharmacol. 2018;83:3-32.

29. Lavrov AV, Ustaeva OA, Adilgereeva EP, Smirnikhina SA, Chelysheva EY, Shukhov OA, Shatokhin YV, Mordanov SV, Turkina AG, Kutsev SI. Copy number variation analysis in cytochromes and glutathione S-transferases may predict efficacy of tyrosine kinase inhibitors in chronic myeloid leukemia. PLoS One. 2017;12(9):e0182901.

30. Roden DM, Wilke RA, Kroemer HK, Stein CM. Pharmacogenomics: the genetics of variable drug responses. Circulation. 2011;123(15):1661-70

31. Stranger BE, Forrest MS, Dunning M, Ingle CE, Beazley C, Thorne N, Redon R, Bird CP, de Grassi A, Lee C, et al. Relative impact of nucleotide and copy number variation on gene expression phenotypes. Science. 2007;315(5813):848-53.

32. Zhao Y, Marotta M, Eichler EE, Eng C, Tanaka H. Linkage disequilibrium between two high-frequency deletion polymorphisms: implications for association studies involving the glutathione-S transferase (GST) genes. PLoS Genet. 2009;5(5):e1000472.

33. Ingelman-Sundberg M, Sim SC, Gomez A, Rodriguez-Antona C. Influence of cytochrome P450 polymorphisms on drug therapies: pharmacogenetic, pharmacoepigenetic and clinical aspects. Pharmacol Ther. 2007;116(3):496-526.
34. Nguyen DQ, Webber C, Ponting CP. Bias of selection on human copynumber variants. PLoS Genet. 2006;2(2):e20.

35. Thorn CF, Ji Y, Weinshilboum RM, Altman RB, Klein TE. PharmGKB summary: very important pharmacogene information for GSTT1. Pharmacogenet Genomics. 2012;22(8):646-51.

36. Economopoulos KP, Sergentanis TN. GSTM1, GSTT1, GSTP1, GSTA1 and colorectal cancer risk: a comprehensive meta-analysis. Eur J Cancer. 2010;46(9):1617-31.

37. Das P, Shaik AP, Bammidi VK. Meta-analysis study of glutathione-Stransferases (GSTM1, GSTP1, and GSTT1) gene polymorphisms and risk of acute myeloid leukemia. Leukemia \& lymphoma. 2009;50(8):1345-51.

38. Kong X, Li Z, Li X. GSTP1, GSTM1, and GSTT1 polymorphisms as predictors of response to chemotherapy in patients with breast cancer: a metaanalysis. Cancer Chemother Pharmacol. 2016;78(6):1163-73.

39. Wang H, Gao X, Zhang X, Gong W, Peng Z, Wang B, Wang L, Chang S, Ma $P$, Wang $S$. Glutathione $S$-transferase gene polymorphisms are associated with an improved treatment response to cisplatin-based chemotherapy in patients with non-small cell lung Cancer (NSCLC): a meta-analysis. Med Sci Monit. 2018;24:7482-92.

40. Tyndale RF, Sellers EM. Variable CYP2A6-mediated nicotine metabolism alters smoking behavior and risk. Drug Metab Dispos. 2001;29(4 Pt 2):548-52.

41. Kamataki T, Fujieda M, Kiyotani K, Iwano S, Kunitoh H. Genetic polymorphism of CYP2A6 as one of the potential determinants of tobaccorelated cancer risk. Biochem Biophys Res Commun. 2005;338(1):306-10.

42. Browning SL, Tarekegn A, Bekele E, Bradman N, Thomas MG. CYP1A2 is more variable than previously thought: a genomic biography of the gene behind the human drug-metabolizing enzyme. Pharmacogenet Genomics. 2010;20(11):647-64.

43. Scott SA, Patel M, Martis S, Lubitz SA, van der Zee S, Yoo C, Edelmann L, Halperin $J$, Desnick RJ. Copy number variation and warfarin dosing: evaluation of CYP2C9, VKORC1, CYP4F2, GGCX and CALU. Pharmacogenomics. 2012;13(3):297-307.

44. Zhou Y, Ingelman-Sundberg M, Lauschke VM. Worldwide distribution of cytochrome P450 alleles: a meta-analysis of population-scale sequencing projects. Clin Pharmacol Ther. 2017;102(4):688-700.

45. Owen RP, Sangkuhl K, Klein TE, Altman RB. Cytochrome P450 2 D6. Pharmacogenet Genomics. 2009;19(7):559-62.

46. Lauschke VM, Ingelman-Sundberg M. Prediction of drug response and adverse drug reactions: from twin studies to next generation sequencing. Eur J Pharm Sci. 2019;130:65-77.

47. Balasopoulou A, Patrinos GP, Katsila T. Pharmacometabolomics informs Viromics toward precision medicine. Front Pharmacol. 2016;7:411.

48. Lakiotaki K, Kanterakis A, Kartsaki E, Katsila T, Patrinos GP. Exploring public genomics data for population pharmacogenomics. PLoS One. 2017;12(8):e0182138.

49. Rodrigues-Soares F, Suarez-Kurtz G. Pharmacogenomics research and clinical implementation in Brazil. Basic Clin Pharmacol Toxicol. 2019;124(5):538-49.

\section{Publisher's Note}

Springer Nature remains neutral with regard to jurisdictional claims in published maps and institutional affiliations.

Ready to submit your research? Choose BMC and benefit from:

- fast, convenient online submission

- thorough peer review by experienced researchers in your field

- rapid publication on acceptance

- support for research data, including large and complex data types

- gold Open Access which fosters wider collaboration and increased citations

- maximum visibility for your research: over $100 \mathrm{M}$ website views per year

At $\mathrm{BMC}$, research is always in progress.

Learn more biomedcentral.com/submission 